\title{
Is soft breaking of BRST symmetry consistent?
}

\author{
Peter Lavrov, ${ }^{a}$ Olaf Lechtenfeld ${ }^{b}$ and Alexander Reshetnyak ${ }^{c}$ \\ ${ }^{a}$ Tomsk State Pedagogical University, \\ Kievskaya St. 60, 634061 Tomsk, Russia \\ ${ }^{b}$ Institut für Theoretische Physik, Leibniz Universität Hannover, \\ Appelstrasse 2, 30167 Hannover, Germany \\ ${ }^{c}$ Institute of Strength Physics and Material Science, \\ Akademicheskii av. 2/4, 634021 Tomsk, Russia \\ E-mail: lavrov@tspu.edu.ru, lechtenf@itp.uni-hannover.de, \\ reshet@ispms.tsc.ru
}

ABSTRACT: A definition of soft breaking of BRST symmetry in the field-antifield formalism is proposed, valid for general gauge theories and arbitrary gauge fixing. The Ward identities for the generating functionals of Green's functions are derived, and their gauge dependence is investigated. We discuss the Gribov-Zwanziger action to the one-parameter family of $R_{\xi}$ gauges. It is argued that gauge theories with a soft breaking of BRST symmetry are inconsistent.

KEYwORDS: Nonperturbative Effects, BRST Quantization, BRST Symmetry

ArXiv EPRINT: 1108.4820 


\section{Contents}

1 Introduction $\quad 1$

2 Soft breaking of BRST symmetry 2

3 Generating functionals and Ward identities 4

$\begin{array}{lll}4 & \text { Gauge dependence } & 7\end{array}$

5 Gribov-Zwanziger action in a one-parameter family of gauges $\quad 10$

6 Conclusions 12

$\begin{array}{ll}\text { A Proof of nilpotency for } \hat{q} & 13\end{array}$

\section{Introduction}

It is well known that BRST symmetry $[1,2]$, as a global fermionic remnant of gauge invariance, plays a fundamental role in quantum field theory, because all fundamental forces existing in Nature can be described in terms of gauge theories [3-6].

Recently, in a series of papers [7-12] based on Zwanziger's action [13, 14], a breakdown of BRST symmetry in Yang-Mills theories has been considered from a new point of view. This breakdown is related to attempts to take into account the Gribov horizon [15], which restricts the domain of integration in the functional integral presenting the Green's functions of the given gauge theory. Effectively this restriction can be implemented by a particular addition to the standard Faddeev-Popov (FP) action. However, this addition is not invariant under the original BRST transformations.

We remark that until now all investigations [7-14] of the Gribov horizon in Yang-Mills theories have been performed in the Landau gauge only. Yet there is a great freedom in the choice of admissible gauges, and it is well known that the Green's functions depend on the gauge. Of course, this dependence is structured, as it must cancel in physical combinations such as the S-matrix. Modern proofs (see, e.g., $[16,17]$ ) of the gauge independence of the S-matrix for Yang-Mills theories are based on BRST symmetry. Any violation of BRST invariance may, therefore, spell doom for the consistency of the gauge theory. Thus, any claim of a breakdown of BRST symmetry warrants serious investigation.

Modern models of the fundamental forces make use of gauge theories more general than Yang-Mills theory. Luckily, the concept of BRST invariance generalizes to supergravity, theories with an open gauge algebra and reducible gauge theories, to name a few. The present paper formulates the soft breaking of BRST symmetry for general gauge theories 
in the field-antifield formalism $[18,19]$. We then investigate the gauge dependence of the Green's functions for arbitrary gauge models with softly broken BRST symmetry.

The paper is organized as follows. In section 2, our definition of the soft breaking of BRST symmetry is given in the field-antifield formalism. Using a suitable regularization scheme, section 3 derives the Ward identities for the customary generating functionals of Green's functions. In section 4 we investigate the dependence of these functionals on an arbitrary gauge, for general gauge theories. A discussion of the Gribov-Zwanziger action for the one-parameter family of $R_{\xi}$ gauges is considered in section 5 . Finally, section 6 gives concluding remarks.

We employ the condensed notation of DeWitt [20]. Derivatives with respect to sources and antifields are taken from the left, while those with respect to fields are taken from the right. Left derivatives with respect to fields are labeled by a subscript $l$. The Grassmann parity of any quantity $A$ is denoted by $\varepsilon(A)$.

\section{Soft breaking of BRST symmetry}

Our starting point is a theory of gauge fields $A^{i}, i=1,2, \ldots, n$, with $\varepsilon\left(A^{i}\right)=\varepsilon_{i}$, described by an initial action $S_{0}=S_{0}(A)$ invariant under the gauge transformations

$$
\delta A^{i}=R_{\alpha}^{i}(A) \xi^{\alpha} \quad \text { hence } \quad S_{0, i}(A) R_{\alpha}^{i}(A)=0 \quad \text { for } \quad \alpha=1,2, \ldots, m, \quad 0<m<n,
$$

parametrized by $m$ arbitrary functions $\xi^{\alpha}$ of the space-time coordinates, with $\varepsilon\left(\xi^{\alpha}\right)=\varepsilon_{\alpha}$. Here, $S_{0, i} \equiv \delta S_{0} / \delta A^{i}$, and $R_{\alpha}^{i}(A)$ are the generators of the gauge transformations, with $\varepsilon\left(R_{\alpha}^{i}\right)=\varepsilon_{i}+\varepsilon_{\alpha}$. We shall not restrict ourselves to some special type of initial gauge theory; it may belong to open gauge theories and/or reducible gauge theories. The type of initial gauge theory defines the structure of configuration space $\left\{\Phi^{A}\right\}$ in the field-antifield formalism $[18,19]$,

$$
\Phi \equiv\left\{\Phi^{A}\right\}=\left\{A^{i}, \ldots\right\} \quad \text { with } \quad \varepsilon\left(\Phi^{A}\right)=\varepsilon_{A},
$$

where the dots indicate the full set of ghost and antighost fields, auxiliary fields and so on. In what follows we do not need to describe the exact structure of the full configuration space. To each field $\Phi^{A}$ of this total configuration space, one introduces the corresponding antifield $\Phi_{A}^{*}$, hence

$$
\Phi^{*} \equiv\left\{\Phi_{A}^{*}\right\}=\left\{A_{i}^{*}, \ldots\right\},
$$

with statistics opposite to that of the corresponding fields $\Phi^{A}$, i.e. $\varepsilon\left(\Phi_{A}^{*}\right)=\varepsilon_{A}+1$.

On the total space of the fields $\Phi^{A}$ and the antifields $\Phi_{A}^{*}$, one defines a bosonic functional $\bar{S}=\bar{S}\left(\Phi, \Phi^{*}\right)$ satisfying the master equation

$$
\frac{1}{2}(\bar{S}, \bar{S})=\mathrm{i} \hbar \Delta \bar{S}
$$

with the boundary condition

$$
\left.\bar{S}\right|_{\Phi^{*}=\hbar=0}=S_{0}(A) .
$$


In (2.4) we used the notation of the antibracket

$$
(F, G) \equiv \frac{\delta F}{\delta \Phi^{A}} \frac{\delta G}{\delta \Phi_{A}^{*}}-(F \leftrightarrow G)(-1)^{[\varepsilon(F)+1] \cdot[\varepsilon(G)+1]}
$$

and of the nilpotent operator

$$
\Delta \equiv(-1)^{\varepsilon_{A}} \frac{\delta_{l}}{\delta \Phi^{A}} \frac{\delta}{\delta \Phi_{A}^{*}} \quad \text { with } \quad \Delta^{2}=0 \quad \text { and } \quad \varepsilon(\Delta)=1 .
$$

We assume that formal manipulations with the operator $\Delta$ can be supported by a suitable regularization scheme. This is a nontrivial requirement, since the operator (2.7) is not well-defined on local functionals. The reason is that for any local functional $F$, one finds that $\Delta F \sim \delta(0)$. The usual way to deal with this problem is to use dimensional regularization [21], which equates $\delta(0)$ to zero. In this paper, we shall imply such a type of regularization, so that the master equation is reduced to the classical master equation

$$
(\bar{S}, \bar{S})=0 .
$$

Using the action $\bar{S}$ and a fermionic gauge fixing functional $\Psi=\Psi(\Phi)$, one can construct the non-degenerate action $S_{e x t}$ by the rule

$$
S_{\text {ext }}\left(\Phi, \Phi^{*}\right)=\bar{S}\left(\Phi, \Phi^{*}+\frac{\delta \Psi}{\delta \Phi}\right) .
$$

This action satisfies the classical master equation

$$
\left(S_{\text {ext }}, S_{\text {ext }}\right)=0
$$

and is used to construct the generating functional of Green's functions in the field-antifield formalism $[18,19]$.

Inspired by $[13,14]$, we modify the action $S_{\text {ext }}$ by adding a functional $M=M\left(\Phi, \Phi^{*}\right)$, defining the full action $S$ as

$$
S=S_{\text {ext }}+M
$$

We shall speak of a soft breaking of BRST symmetry in the field-antifield formalism if the condition

$$
(M, M)=0
$$

is fulfilled. Therefore, the basic classical equation of our approach to the soft breaking of BRST symmetry reads

$$
\frac{1}{2}(S, S)=(S, M)
$$

If the soft breaking of BRST symmetry originates from a modification of the integration measure, then $M$ will be a functional of the field variables $\Phi^{A}$ only, i.e. $M=M(\Phi)$. In this case, the condition (2.12) is automatically valid. In fact, this is exactly the situation for Yang-Mills theory in Landau gauge, when one takes into account the Gribov horizon [13, 14]. We do not restrict ourselves to this special case and consider the more general situation of $M=M\left(\Phi, \Phi^{*}\right)$. 
It is interesting to note that the right-hand side of the basic classical equation (2.13) can be presented in the form

$$
(S, M)=\hat{s} M,
$$

where $\hat{s}$ denotes the Slavnov-Taylor operator defined by the rule

$$
\hat{s}=\left(S_{\text {ext }}, \bullet\right) .
$$

Due to (2.10) this operator is nilpotent,

$$
\hat{s}^{2}=0
$$

and we find that

$$
\hat{s}(S, S)=0 .
$$

On this level we formally meet the same relation as for general gauge theories without a soft breaking of BRST symmetry.

\section{Generating functionals and Ward identities}

Let us consider some quantum consequences of the classical equations (2.10), (2.12) and (2.13). To this end we introduce the generating functional of Green's functions,

$$
Z\left(J, \Phi^{*}\right)=\int D \Phi \exp \left\{\frac{\mathrm{i}}{\hbar}\left(S\left(\Phi, \Phi^{*}\right)+J_{A} \Phi^{A}\right)\right\}
$$

where $S\left(\Phi, \Phi^{*}\right)$ satisfies the basic classical equation (2.13) and has the form (2.11). Furthermore, $J_{A}$ are the usual external sources for the fields $\Phi^{A}$. The Grassmann parities of these sources are defined in a natural way, $\varepsilon\left(J_{A}\right)=\varepsilon_{A}$.

From (2.10) it follows that

$$
0=\int D \Phi \frac{\delta S_{\text {ext }}}{\delta \Phi^{A}} \frac{\delta S_{\text {ext }}}{\delta \Phi_{A}^{*}} \exp \left\{\frac{\mathrm{i}}{\hbar}\left(S_{e x t}+M+J_{A} \Phi^{A}\right)\right\} .
$$

Performing the usual manipulations with the functional integral we arrive at the following identity for the generating functional $Z$,

$$
\frac{\hbar}{\mathrm{i}}\left(J_{A}+M_{A}\left(\frac{\hbar}{\mathrm{i}} \frac{\delta}{\delta J}, \Phi^{*}\right)\right) \frac{\delta Z\left(J, \Phi^{*}\right)}{\delta \Phi_{A}^{*}}-J_{A} M^{A *}\left(\frac{\hbar}{\mathrm{i}} \frac{\delta}{\delta J}, \Phi^{*}\right) Z\left(J, \Phi^{*}\right)=0 .
$$

Here the notations

$$
\left.M_{A}\left(\frac{\hbar}{\mathrm{i}} \frac{\delta}{\delta J}, \Phi^{*}\right) \equiv \frac{\delta M\left(\Phi, \Phi^{*}\right)}{\delta \Phi^{A}}\right|_{\Phi \rightarrow \frac{\hbar}{\mathrm{i}} \frac{\delta}{\delta J}} \quad \text { and }\left.\quad M^{A *}\left(\frac{\hbar}{\mathrm{i}} \frac{\delta}{\delta J}, \Phi^{*}\right) \equiv \frac{\delta M\left(\Phi, \Phi^{*}\right)}{\delta \Phi_{A}^{*}}\right|_{\Phi \rightarrow \frac{\hbar}{\mathrm{i}} \frac{\delta}{\delta J}}
$$

have been used. In case of $M=0$, the identity (3.2) is reduced to the usual Ward identity for the generating functional of Green's functions in the field-antifield formalism. Hence, we refer to (3.2) as the Ward identity for $Z$ in a gauge theory with softly broken BRST symmetry. 
Introducing the generating functional of connected Green's functions,

$$
W\left(J, \Phi^{*}\right)=-\mathrm{i} \hbar \ln Z\left(J, \Phi^{*}\right),
$$

the identity (3.2) can be rewritten as

$$
\left(J_{A}+M_{A}\left(\frac{\delta W}{\delta J}+\frac{\hbar}{\mathrm{i}} \frac{\delta}{\delta J}, \Phi^{*}\right)\right) \frac{\delta W\left(J, \Phi^{*}\right)}{\delta \Phi_{A}^{*}}-J_{A} M^{A *}\left(\frac{\delta W}{\delta J}+\frac{\hbar}{\mathrm{i}} \frac{\delta}{\delta J}, \Phi^{*}\right)=0 .
$$

The generating functional of the vertex functions or effective action is obtained by Legendre transforming $W$,

$$
\Gamma\left(\Phi, \Phi^{*}\right)=W\left(J, \Phi^{*}\right)-J_{A} \Phi^{A} \quad \text { where } \quad \Phi^{A}=\frac{\delta W}{\delta J_{A}} \quad \text { and } \quad \frac{\delta \Gamma}{\delta \Phi^{A}}=-J_{A} .
$$

Taking into account the equality

$$
\frac{\delta \Gamma}{\delta \Phi_{A}^{*}}=\frac{\delta W}{\delta \Phi_{A}^{*}},
$$

we can rewrite the identity (3.4) in terms of $\Gamma$ as

$$
\frac{1}{2}(\Gamma, \Gamma)=\frac{\delta \Gamma}{\delta \Phi^{A}} \widehat{M}^{A *}+\widehat{M}_{A} \frac{\delta \Gamma}{\delta \Phi_{A}^{*}} .
$$

Here, we have used the notation

$$
\left.\widehat{M}_{A} \equiv \frac{\delta M\left(\Phi, \Phi^{*}\right)}{\delta \Phi^{A}}\right|_{\Phi \rightarrow \widehat{\Phi}} \quad \text { and }\left.\quad \widehat{M}^{A *} \equiv \frac{\delta M\left(\Phi, \Phi^{*}\right)}{\delta \Phi_{A}^{*}}\right|_{\Phi \rightarrow \widehat{\Phi}}
$$

where

$$
\widehat{\Phi}^{A}=\Phi^{A}+\mathrm{i} \hbar\left(\Gamma^{\prime \prime}-1\right)^{A B} \frac{\delta_{l}}{\delta \Phi^{B}}
$$

and the matrix $\left(\Gamma^{\prime \prime}-1\right)$ is inverse to the matrix $\Gamma^{\prime \prime}$ with elements

$$
\left(\Gamma^{\prime \prime}\right)_{A B}=\frac{\delta_{l}}{\delta \Phi^{A}}\left(\frac{\delta \Gamma}{\delta \Phi^{B}}\right), \quad \text { i.e. } \quad\left(\Gamma^{\prime \prime}-1\right)^{A C}\left(\Gamma^{\prime \prime}\right)_{C B}=\delta_{B}^{A} .
$$

Again, in the case $M=0$ the identity (3.6) coincides with the Ward identity for the effective action in the field-antifield formalism. Note that the identity (3.6) is compatible with the basic classical equation (2.13), since $\hbar \rightarrow 0$ yields $\Gamma=S, \widehat{M}=M$, and (3.6) is reduced to (2.13).

But this is not the end of the story, because on the classical level we also have the restriction $(2.12)$, i.e. $(M, M)=0$, which on the quantum level generates an additional identity. To derive it we consider a direct consequence of (2.12),

$$
0=\int D \Phi \frac{\delta M}{\delta \Phi^{A}} \frac{\delta M}{\delta \Phi_{A}^{*}} \exp \left\{\frac{\mathrm{i}}{\hbar}\left(S_{e x t}+M+J_{A} \Phi^{A}\right)\right\} .
$$

Omitting the details of the functional-integral manipulations, we can rewrite (3.10) as

$$
\widehat{M}_{A} \widehat{M}^{A *}=0 .
$$


In addition, the relations $\Delta M=0$ and $\Delta S=0$ yield quantum consequences. Indeed, from the evident equality

$$
0=\int D \Phi(-1)^{\varepsilon_{A}} \frac{\delta_{l}}{\delta \Phi^{A}}\left[\frac{\delta M}{\delta \Phi_{A}^{*}} \exp \left\{\frac{\mathrm{i}}{\hbar}\left(S+J_{A} \Phi^{A}\right)\right\}\right]
$$

it follows that

$$
0=\int D \Phi\left[\Delta M+\frac{\mathrm{i}}{\hbar}\left(J_{A}+\frac{\delta S}{\delta \Phi^{A}}\right) \frac{\delta M}{\delta \Phi_{A}^{*}}\right] \exp \left\{\frac{\mathrm{i}}{\hbar}\left(S+J_{A} \Phi^{A}\right)\right\},
$$

which produces the identity

$$
\left(\frac{\delta \Gamma}{\delta \Phi^{A}}-\widehat{S}_{A}\right) \widehat{M}^{A *}=0
$$

where

$$
\widehat{S}_{A} \equiv S_{A}\left(\widehat{\Phi}, \Phi^{*}\right)=\left.\frac{\delta S\left(\Phi, \Phi^{*}\right)}{\delta \Phi^{A}}\right|_{\Phi \rightarrow \widehat{\Phi}} \quad \text { and } \quad \widehat{\Phi}^{A}=\Phi^{A}+\mathrm{i} \hbar\left(\Gamma^{\prime \prime}-1\right)^{A B} \frac{\delta_{l}}{\delta \Phi^{B}} .
$$

In turn starting with the equality

$$
0=\int D \Phi(-1)^{\varepsilon_{A}} \frac{\delta_{l}}{\delta \Phi^{A}}\left[\frac{\delta S}{\delta \Phi_{A}^{*}} \exp \left\{\frac{\mathrm{i}}{\hbar}\left(S+J_{A} \Phi^{A}\right)\right\}\right]
$$

we have

$$
0=\int D \Phi\left[\Delta S+\frac{\mathrm{i}}{\hbar}\left(J_{A}+\frac{\delta S}{\delta \Phi^{A}}\right) \frac{\delta S}{\delta \Phi_{A}^{*}}\right] \exp \left\{\frac{\mathrm{i}}{\hbar}\left(S+J_{A} \Phi^{A}\right)\right\},
$$

which gives us

$$
\left(\frac{\delta \Gamma}{\delta \Phi^{A}}-\widehat{S}_{A}\right) \frac{\delta \Gamma}{\delta \Phi_{A}^{*}}=0
$$

as the quantum version of the equality $\Delta S=0$.

Finally, it should be noted that from the equality

$$
0=\int D \Phi \frac{\delta}{\delta \Phi^{A}}\left[\exp \left\{\frac{\mathrm{i}}{\hbar}\left(S+J_{A} \Phi^{A}\right)\right\}\right]
$$

we can derive in the usual manner the relation

$$
\frac{\delta \Gamma}{\delta \Phi^{A}}=\widehat{S}_{A}
$$

which is nothing but the other representation of equation for $\Gamma$ (see (3.5)). It implies the relations (3.12) and (3.14).

Analogously, starting with the identity

$$
\frac{\delta F(\Phi)}{\delta \Phi_{A}^{*}} \equiv 0
$$

for an arbitrary functional $F(\Phi)$, we get

$$
0=\int D \Phi \frac{\delta F(\Phi)}{\delta \Phi_{A}^{*}} \exp \left\{\frac{\mathrm{i}}{\hbar}\left(S+J_{A} \Phi^{A}\right)\right\}
$$


and therefore

$$
F(\widehat{\Phi}) \frac{\delta \Gamma}{\delta \Phi_{A}^{*}}=F(\widehat{\Phi}) S^{A *}\left(\widehat{\Phi}, \Phi^{*}\right) .
$$

Since the functional $F(\Phi)$ was arbitrary, we also have the relation

$$
\frac{\delta \Gamma}{\delta \Phi_{A}^{*}}=\widehat{S}^{A *}
$$

with

$$
\widehat{S}^{A *} \equiv S^{A *}\left(\widehat{\Phi}, \Phi^{*}\right)=\left.\frac{\delta S\left(\Phi, \Phi^{*}\right)}{\delta \Phi_{A}^{*}}\right|_{\Phi \rightarrow \widehat{\Phi}} .
$$

Clearly, in the $\hbar \rightarrow 0$ limit $\widehat{M}=M$, and the identity (3.11) is reduced to (2.12). Therefore, we have a full set of equalities which describe on the classical and quantum level general gauge theories with a soft breaking of BRST symmetry in arbitrary gauges within the field-antifield formalism.

\section{Gauge dependence}

We turn to a discussion of gauge dependence of the generating functionals $Z, W$ and $\Gamma$ for general gauge theories with a soft breaking of BRST symmetry as defined in the previous section. The derivation of this dependence is based on the fact that any variation of the gauge-fixing functional, $\Psi(\Phi) \rightarrow \Psi(\Phi)+\delta \Psi(\Phi)$, leads to a variation of the action $S_{\text {ext }}(2.9)$ and the functional $Z$ [22]. The variation of $S_{\text {ext }}$ can be presented in the form

$$
\delta S_{e x t}=\frac{\delta \delta \Psi}{\delta \Phi^{A}} \frac{\delta S_{\text {ext }}}{\delta \Phi_{A}^{*}}
$$

or as

$$
\delta S_{\text {ext }}=-\left(S_{\text {ext }}, \delta \Psi\right)=-\hat{s} \delta \Psi .
$$

We also allow the functional $M$ to be gauge dependent, with $\delta M\left(\Phi, \Phi^{*}\right)$ being its variation simultaneous to the variation $\delta \Psi$ of the gauge-fixing functional. From (3.1), (4.1) and the variation of $M$ we obtain the gauge variation of $Z$,

$$
\delta Z\left(J, \Phi^{*}\right)=\frac{\mathrm{i}}{\hbar} \int D \Phi\left(\frac{\delta \delta \Psi}{\delta \Phi^{A}} \frac{\delta S_{\text {ext }}}{\delta \Phi_{A}^{*}}+\delta M\right) \exp \left\{\frac{\mathrm{i}}{\hbar}\left(S\left(\Phi, \Phi^{*}\right)+J_{A} \Phi^{A}\right)\right\} .
$$

With the help of

$$
\begin{aligned}
0 & =\int D \Phi \frac{\delta_{l}}{\delta \Phi^{A}}\left[\delta \Psi \frac{\delta S_{\text {ext }}}{\delta \Phi_{A}^{*}} \exp \left\{\frac{\mathrm{i}}{\hbar}\left(S\left(\Phi, \Phi^{*}\right)+J_{A} \Phi^{A}\right)\right\}\right] \\
& =\int D \Phi\left[\frac{\delta \delta \Psi}{\delta \Phi^{A}} \frac{\delta S_{\text {ext }}}{\delta \Phi_{A}^{*}}-\frac{\mathrm{i}}{\hbar}\left(J_{A}+\frac{\delta S}{\delta \Phi^{A}}\right) \frac{\delta S_{\text {ext }}}{\delta \Phi_{A}^{*}} \delta \Psi\right] \exp \left\{\frac{\mathrm{i}}{\hbar}\left(S\left(\Phi, \Phi^{*}\right)+J_{A} \Phi^{A}\right)\right\},
\end{aligned}
$$

where $\Delta S_{\text {ext }}=0$ was used, and the relation

$$
\frac{\delta S}{\delta \Phi^{A}} \frac{\delta S_{e x t}}{\delta \Phi_{A}^{*}}=\frac{\delta M}{\delta \Phi^{A}} \frac{\delta S}{\delta \Phi_{A}^{*}},
$$


we can rewrite (4.3) as

$$
\begin{aligned}
\delta Z\left(J, \Phi^{*}\right)=\frac{\mathrm{i}}{\hbar}[ & \left(J_{A}+M_{A}\left(\frac{\hbar}{\mathrm{i}} \frac{\delta}{\delta J}, \Phi^{*}\right)\right) \frac{\delta}{\delta \Phi_{A}^{*}} \delta \Psi\left(\frac{\hbar}{\mathrm{i}} \frac{\delta}{\delta J}\right) \\
& \left.\quad-\frac{\mathrm{i}}{\hbar} J_{A} M^{A *}\left(\frac{\hbar}{\mathrm{i}} \frac{\delta}{\delta J}, \Phi^{*}\right) \delta \Psi\left(\frac{\hbar}{\mathrm{i}} \frac{\delta}{\delta J}\right)+\delta M\left(\frac{\hbar}{\mathrm{i}} \frac{\delta}{\delta J}, \Phi^{*}\right)\right] Z\left(J, \Phi^{*}\right) \\
= & \frac{\mathrm{i}}{\hbar}\left[\hat{q} \delta \Psi\left(\frac{\hbar}{\mathrm{i}} \frac{\delta}{\delta J}\right)+\delta M\left(\frac{\hbar}{\mathrm{i}} \frac{\delta}{\delta J}, \Phi^{*}\right)\right] Z\left(J, \Phi^{*}\right),
\end{aligned}
$$

where we have abbreviated the first line by introducing the nilpotent fermionic operator

$$
\hat{q}=\left(J_{A}+M_{A}\left(\frac{\hbar}{\mathrm{i}} \frac{\delta}{\delta J}, \Phi^{*}\right)\right) \frac{\delta}{\delta \Phi_{A}^{*}}-\frac{\mathrm{i}}{\hbar} J_{A} M^{A *}\left(\frac{\hbar}{\mathrm{i}} \frac{\delta}{\delta J}, \Phi^{*}\right) .
$$

Its nilpotency, $\hat{q}^{2}=0$, is proved in the appendix.

The corresponding variation of the generating functional of connected Green's functions takes the form

$$
\delta W\left(J, \Phi^{*}\right)=\frac{\hbar}{\mathrm{i}} Z^{-1} \delta Z=\hat{Q} \delta \Psi\left(\frac{\delta W}{\delta J}+\frac{\hbar}{\mathrm{i}} \frac{\delta}{\delta J}\right)+\delta M\left(\frac{\delta W}{\delta J}+\frac{\hbar}{\mathrm{i}} \frac{\delta}{\delta J}, \Phi^{*}\right),
$$

where the fermionic operator $\hat{Q}$ is unitarily related to $\hat{q}$,

$$
\hat{Q}=\exp \left\{-\frac{\mathrm{i}}{\hbar} W\right\} \hat{q} \exp \left\{\frac{\mathrm{i}}{\hbar} W\right\}=\left(J_{A}+M_{A}\left(\frac{\delta W}{\delta J}+\frac{\hbar}{\mathrm{i}} \frac{\delta}{\delta J}, \Phi^{*}\right)\right) \frac{\delta}{\delta \Phi_{A}^{*}},
$$

with the help of the Ward identity (3.4). Note that all terms in $\hat{Q}$ contain an antifield derivative. From its construction, $\hat{Q}$ is nilpotent as well, i.e. $\hat{Q}^{2}=0$.

Let us proceed to the gauge variation of the effective action. We firstly note that $\delta \Gamma=\delta W$. Secondly, we observe that the definitions (3.5) and the Ward identity (3.6) imply that

$$
\left.\frac{\delta}{\delta \Phi^{*}}\right|_{J}=\left.\frac{\delta}{\delta \Phi^{*}}\right|_{\Phi}+\left.\frac{\delta \Phi}{\delta \Phi^{*}} \frac{\delta_{l}}{\delta \Phi}\right|_{\Phi^{*}} .
$$

Next, differentiating the Ward identities (3.2) with respect to the sources $J$, then rewriting these relations for the functional $W$ and transforming the latter with allowance for (3.5) and (3.6), we arrive at

$$
\begin{aligned}
\left.\hat{Q} \Phi^{A}\right|_{J}= & \left(\widehat{M}^{A *}-\frac{\delta \Gamma}{\delta \Phi_{A}^{*}}\right)(-1)^{\varepsilon_{A}} \\
& +\frac{\mathrm{i}}{\hbar}\left(\Phi^{A} \widehat{M}_{B} \frac{\delta \Gamma}{\delta \Phi_{B}^{*}}(-1)^{\varepsilon_{A}}-\widehat{M}_{B} \frac{\delta \Gamma}{\delta \Phi_{B}^{*}} \Phi^{A}\right) \\
& +\frac{\mathrm{i}}{\hbar}\left(\Phi^{A} \frac{\delta \Gamma}{\delta \Phi^{B}} \widehat{M}^{B^{*}}(-1)^{\varepsilon_{A}}-\frac{\delta \Gamma}{\delta \Phi^{B}} \widehat{M}^{B^{*}} \Phi^{A}\right) .
\end{aligned}
$$

From (4.7)-(4.10) we can represent the gauge variation of the effective action in the following form,

$$
\delta \Gamma=\hat{s}_{q}\langle\delta \Psi\rangle+\langle\delta M\rangle,
$$


where the operator $\hat{s}_{q}$ is given by

$$
\begin{aligned}
\hat{s}_{q}= & -(\Gamma, \bullet)+\widehat{M}_{A} \frac{\delta}{\delta \Phi_{A}^{*}}+(-1)^{\varepsilon_{A}} \widehat{M}^{A *} \frac{\delta_{l}}{\delta \Phi^{A}} \\
& -\frac{\mathrm{i}}{\hbar}\left(\widehat{M}_{B} \frac{\delta \Gamma}{\delta \Phi_{B}^{*}} \Phi^{A}-(-1)^{\varepsilon_{A}} \Phi^{A} \widehat{M}_{B} \frac{\delta \Gamma}{\delta \Phi_{B}^{*}}\right) \frac{\delta_{l}}{\delta \Phi^{A}} \\
& -\frac{\mathrm{i}}{\hbar}\left(\frac{\delta \Gamma}{\delta \Phi^{B}} \widehat{M}^{B *} \Phi^{A}-(-1)^{\varepsilon_{A}} \Phi^{A} \frac{\delta \Gamma}{\delta \Phi^{B}} \widehat{M}^{B *}\right) \frac{\delta_{l}}{\delta \Phi^{A}}
\end{aligned}
$$

and we introduced the notation

$$
\langle\delta \Psi\rangle=\delta \Psi(\widehat{\Phi}) \cdot 1 \quad \text { and } \quad\langle\delta M\rangle=\delta M\left(\widehat{\Phi}, \Phi^{*}\right) \cdot 1 .
$$

Because $\hat{s}_{q}$ is related to $\hat{Q}$ via a Legendre transformation (which is a change of variables), it must be nilpotent as well, $\hat{s}_{q}^{2}=0$.

Another extremely useful representation for $\delta \Gamma$ is obtained by a slightly different rewriting of (4.10) as follows,

$$
\begin{aligned}
\frac{\delta \Gamma}{\delta \Phi_{A}^{*}}-\widehat{M}^{A *}= & -(-1)^{\varepsilon_{A} \varepsilon_{B}}\left(\widehat{M}_{B}-\frac{\delta \Gamma}{\delta \Phi^{B}}\right)\left(\Gamma^{\prime \prime}-1\right)^{A C} \frac{\delta_{l}}{\delta \Phi^{C}} \frac{\delta \Gamma}{\delta \Phi_{B}^{*}} \\
& +\frac{\mathrm{i}}{\hbar}\left(\Phi^{A} \widehat{M}_{B} \frac{\delta \Gamma}{\delta \Phi_{B}^{*}}-(-1)^{\varepsilon_{A}} \widehat{M}_{B} \frac{\delta \Gamma}{\delta \Phi_{B}^{*}} \Phi^{A}\right) \\
& +\frac{\mathrm{i}}{\hbar}\left(\Phi^{A} \frac{\delta \Gamma}{\delta \Phi^{B}} \widehat{M}^{B *}-(-1)^{\varepsilon_{A}} \frac{\delta \Gamma}{\delta \Phi^{B}} \widehat{M}^{B *} \Phi^{A}\right) .
\end{aligned}
$$

As the result we obtain our final expression for the gauge variation of the effective action,

$$
\delta \Gamma=\frac{\delta \Gamma}{\delta \Phi^{A}} \widehat{F}^{A}\langle\delta \Psi\rangle-\widehat{M}_{A} \widehat{F}^{A}\langle\delta \Psi\rangle+\langle\delta M\rangle,
$$

with the operator definition

$$
\widehat{F}^{A}=-\frac{\delta}{\delta \Phi_{A}^{*}}-(-1)^{\varepsilon_{B}\left(\varepsilon_{A}+1\right)}\left(\Gamma^{\prime \prime}-1\right)^{B C}\left(\frac{\delta_{l}}{\delta \Phi^{C}} \frac{\delta \Gamma}{\delta \Phi_{A}^{*}}\right) \frac{\delta_{l}}{\delta \Phi^{B}} .
$$

We see from (4.15) that on shell the effective action is generally gauge dependent since

$$
\frac{\delta \Gamma}{\delta \Phi^{A}}=0 \quad \longrightarrow \quad \delta \Gamma \neq 0
$$

This negates a consistent formulation of a soft breaking of BRST symmetry within the field-antifield formalism, unless perhaps the two last terms in (4.15) cancel each other,

$$
\langle\delta M\rangle=\widehat{M}_{A} \widehat{F}^{A}\langle\delta \Psi\rangle .
$$

This is a severe restriction on the BRST-breaking functional $M$ for the effective action to be gauge independent on-shell. The same statement is valid for physical S-matrix. In fact, (4.18) fixes the gauge variation of $M=M\left(\Phi, \Phi^{*}\right)$ under a change of the gauge-fixing functional $\Psi$ to be

where (see (2.11) and (2.13))

$$
\delta M=\frac{\delta M}{\delta \Phi^{A}} \widehat{F}_{0}^{A} \delta \Psi
$$

$$
\widehat{F}_{0}^{A}=-(-1)^{\varepsilon_{B}\left(\varepsilon_{A}+1\right)}\left(S^{\prime \prime}-1\right)^{B C}\left(\frac{\delta_{l}}{\delta \Phi^{C}} \frac{\delta S}{\delta \Phi_{A}^{*}}\right) \frac{\delta_{l}}{\delta \Phi^{B}} .
$$




\section{Gribov-Zwanziger action in a one-parameter family of gauges}

In this section we shall apply our above-described general consideration of a soft BRST breaking to the important case of Yang-Mills theories, since those had been the subject of recent investigations [7-12]. The initial classical action $S_{0}$ of Yang-Mills fields $A_{\mu}^{a}(x)$, which take values in the adjoint representation of $s u(N)$ so that, $a=1, \ldots, N^{2}-1$, has the standard form

$$
S_{0}(A)=-\frac{1}{4} \int \mathrm{d}^{D} x F_{\mu \nu}^{a} F^{\mu \nu a} \quad \text { with } \quad F_{\mu \nu}^{a}=\partial_{\mu} A_{\nu}^{a}-\partial_{\nu} A_{\mu}^{a}+f^{a b c} A_{\mu}^{b} A_{\nu}^{c},
$$

where $\mu, \nu=0,1, \ldots, D-1$, the Minkowski space has signature $(-,+, \ldots,+)$, and $f^{a b c}$ denote the (totally antisymmetric) structure constants of the Lie algebra $s u(N)$. The action (5.1) is invariant under the gauge transformations

$$
\delta A_{\mu}^{a}=D_{\mu}^{a b} \xi^{b} \quad \text { with } \quad D_{\mu}^{a b}=\delta^{a b} \partial_{\mu}+f^{a c b} A_{\mu}^{c} .
$$

The field configuration space of Yang-Mills theory,

$$
\left\{\Phi^{A}\right\}=\left\{A_{\mu}^{a}, B^{a}, C^{a}, \bar{C}^{a}\right\} \quad \text { with } \quad \varepsilon\left(C^{a}\right)=\varepsilon\left(\bar{C}^{a}\right)=1, \quad \varepsilon\left(A_{\mu}^{a}\right)=\varepsilon\left(B^{a}\right)=0,
$$

includes the (scalar) Faddeev-Popov ghost and antighost fields $C^{a}$ and $\bar{C}^{a}$, respectively, as well as the Nakanishi-Lautrup auxiliary fields $B^{a}$. The corresponding set of antifields is

$$
\left\{\Phi_{A}^{*}\right\}=\left\{A^{* a \mu}, B^{* a}, C^{* a}, \bar{C}^{* a}\right\} \quad \text { with } \quad \varepsilon\left(A^{* a \mu}\right)=\varepsilon\left(B^{* a}\right)=1, \quad \varepsilon\left(C^{* a}\right)=\varepsilon\left(\bar{C}^{* a}\right)=0 .
$$

A solution to the classical master equation (2.8) can be presented in the form

$$
\bar{S}\left(\Phi, \Phi^{*}\right)=S_{0}(A)+A^{* a \mu} D_{\mu}^{a b} C^{b}+\frac{1}{2} C^{* a} f^{a b c} C^{b} C^{c}+\bar{C}^{* a} B^{a} .
$$

The gauge-fixing functional can be chosen as

$$
\Psi(\Phi)=\bar{C}^{a} \chi^{a}(A, B)
$$

with free bosonic functions $\chi^{a}$, so that the non-degenerate action $S_{\text {ext }}(2.9)$ becomes

$$
\begin{aligned}
S_{e x t}\left(\Phi, \Phi^{*}\right) & =S_{0}(A)+\left(A^{* a \mu}+\bar{C}^{c} \frac{\delta \chi^{c}}{\delta A_{\mu}^{a}}\right) D_{\mu}^{a b} C^{b}+\frac{1}{2} C^{* a} f^{a b c} C^{b} C^{c}+\left(\bar{C}^{* a}+\chi^{a}\right) B^{a} \\
& =S_{F P}(\Phi)+A^{* a \mu} D_{\mu}^{a b} C^{b}+\frac{1}{2} C^{* a} f^{a b c} C^{b} C^{c}+\bar{C}^{* a} B^{a}
\end{aligned}
$$

where $S_{F P}(\Phi)$ is the Faddeev-Popov action

$$
S_{F P}(\Phi)=S_{0}(A)+\bar{C}^{a} K^{a b} C^{b}+\chi^{a} B^{a} \quad \text { with } \quad K^{a b}=\frac{\delta \chi^{a}}{\delta A_{\mu}^{c}} D_{\mu}^{c b} .
$$

The actions (5.8) and (5.7) are invariant under the BRST transformation

$$
\delta_{B} A_{\mu}^{a}=D_{\mu}^{a b} C^{b} \theta, \quad \delta_{B} \bar{C}^{a}=B^{a} \theta, \quad \delta_{B} B^{a}=0, \quad \delta_{B} C^{a}=\frac{1}{2} f^{a b c} C^{b} C^{c} \theta
$$

where $\theta$ is a constant Grassmann parameter. 
In $[13,14]$ it has been shown that the Gribov horizon [15] in Yang-Mills theory (5.1) in the Landau gauge,

$$
\chi^{a}(A, B)=\partial^{\mu} A_{\mu}^{a} \quad \longrightarrow \quad K^{a b}=\partial^{\mu} D_{\mu}^{a b},
$$

can be taken in to account by adding to the Faddeev-Popov action (5.8) the non-local functional $^{1}$

$$
M(A)=\gamma^{2}\left(f^{a b c} A_{\mu}^{b}\left(K^{-1}\right)^{a d} f^{d e c} A^{e \mu}+D\left(N^{2}-1\right)\right),
$$

where $K^{-1}$ is the matrix inverse to the Faddeev-Popov operator $K^{a b}$ in (5.10). The socalled thermodynamic or Gribov parameter $\gamma$ is determined in a self-consistent way by the gap equation $[13,14]$

$$
\frac{\partial \mathcal{E}_{v a c}}{\partial \gamma}=0
$$

where $\mathcal{E}_{\text {vac }}$ is the vacuum energy given by

$$
\exp \left\{\frac{\mathrm{i}}{\hbar} \mathcal{E}_{\text {vac }}\right\}=\int D \Phi \exp \left\{\frac{\mathrm{i}}{\hbar} S_{G Z}(\Phi)\right\}
$$

pertaining to the Gribov-Zwanziger action [7-12]

$$
S_{G Z}(\Phi)=S_{F P}(\Phi)+M(A) .
$$

Note that the functional $M(A)$ in (5.11) is not invariant under the BRST transformation (5.9) but trivially satisfies the condition (2.12) of soft BRST breaking because of its independence on antifields.

The Gribov-Zwanziger action was intensively investigated in a series of papers [7-12] where various quantum properties of gauge models with this action have been studied. We stress however that it was impossible in principle to establish the gauge independence of physical quantities in these theories because they were formulated in the Landau gauge (5.10) only. Here, we are going to clarify this crucial issue.

To this end, we extend the Gribov-Zwanziger action (5.14) to the one-parameter family of $R_{\xi}$ gauges,

$$
\chi^{a}(A, B, \xi)=\partial^{\mu} A_{\mu}^{a}+\frac{\xi}{2} B^{a}
$$

with a real parameter $\xi$ interpolating between the Landau gauge $(\xi=0)$ and the Feynman gauge $(\xi=1)$. The Faddeev-Popov action is then written as

$$
S_{F P}(\Phi, \xi)=S_{0}(A)+\bar{C}^{a} \partial^{\mu} D_{\mu}^{a b} C^{b}+\left(\partial^{\mu} A_{\mu}^{a}\right) B^{a}+\frac{\xi}{2} B^{a} B^{a} .
$$

The Faddeev-Popov operator $K^{a b}$ is obviously independent of $\xi$, but the functional $M$ must be modified away from $\xi=0$, already because $K^{a b}$ ceases to be hermitian [23]. Although a suitable functional $M(A, B, \xi)$ is not known, we assume its existence with

$$
\lim _{\xi \rightarrow 0} M(A, B, \xi)=M(A)
$$

\footnotetext{
${ }^{1}$ The choice of [7-12] agrees with ours after Wick rotation, integrating out auxiliary fields and renaming $\gamma^{4} \rightarrow \gamma^{2}$
} 
where $M(A)$ is given by (5.11). Now we propose the Gribov-Zwanziger action for YangMills theories (5.1) in the $R_{\xi}$ gauge family (5.15) as

$$
S_{G Z}(\Phi, \xi)=S_{F P}(\Phi, \xi)+M(A, B, \xi)
$$

Because the BRST transformation (5.9) does not depend on the gauge fixing, from (5.17) by continuity we can conclude that

$$
\delta_{B} M(A, B, \xi) \neq 0 \quad \longrightarrow \quad \delta_{B} S_{G Z}(\Phi, \xi) \neq 0
$$

Let us recall our consistency condition (4.19), which takes the form

$$
\delta M(A, B, \xi) \stackrel{!}{=} \frac{1}{2} \frac{\delta M(A, B, \xi)}{\delta \Phi^{A}} \widehat{F}_{0}^{A} \bar{C}^{a} B^{a} \delta \xi
$$

Since the right-hand side necessarily depends on the ghost, antighost or auxiliary fields, it cannot match the left-hand side for our choice of $M$. Therefore, soft breaking of BRST symmetry is not consistent in $R_{\xi}$ gauges.

\section{Conclusions}

We have proposed a definition of soft breaking of BRST symmetry in the field-antifield formalism. To this end, a 'breaking functional' $M$ had to be added to the gauge-fixed action $S_{\text {ext }}$. The latter is constructed from an arbitrary classical gauge-invariant action $S_{0}$ with the rules of the field-antifield method. In terms of the functional $M$, the soft breaking of BRST symmetry was defined by the analog of the classical master equation $(M, M)=0$. We have derived all Ward identities for the generating functional of Green's functions, of connected Green's functions and of vertex functions, denoted by $Z, W$ and $\Gamma$, respectively. These identities were employed to investigate the gauge dependence of those functionals. It was shown that $\Gamma$ as well as the S-matrix are on-shell gauge dependent in general. We discussed the Gribov-Zwanziger action for the one-parameter family of $R_{\xi}$ gauges. Already in this simple case, the functional $\Gamma$ turned out to depend on the gauge even on shell. We are forced to conclude that a consistent quantization of gauge theories with a soft breaking of BRST symmetry does not exist.

\section{Acknowledgments}

The authors thank I.L. Buchbinder and I.V. Tyutin for useful discussions. P.L. is grateful to the Institute for Theoretical Physics of Leibniz Universität Hannover for warm hospitality. We are grateful to various authors of [7-12] for a critical assessment of earlier versions. This work was partially supported by the joint DFG grant 436 RUS 113/669/4. The work of P.L. is also supported by the LRSS grant 3558.2010.2, the RFBR-Ukraine grant 11-02-90445 and the RFBR grant 09-02-00078. 


\section{A Proof of nilpotency for $\hat{q}$}

For simplicity of writing let us abbreviate

$$
M_{A}\left(\frac{\hbar}{\mathrm{i}} \frac{\delta}{\delta J}, \Phi^{*}\right)=: M_{A} \quad \text { and } \quad M^{A *}\left(\frac{\hbar}{\mathrm{i}} \frac{\delta}{\delta J}, \Phi^{*}\right)=: M^{A *} .
$$

The square of $\hat{q}$ may be directly presented as a sum of four operators,

$$
\begin{aligned}
\hat{q}^{2}= & {\left[\left(J_{A}+M_{A}\right) \frac{\delta}{\delta \Phi_{A}^{*}}-\frac{\mathrm{i}}{\hbar} J_{A} M^{A *}\right]^{2} \equiv \sum_{i=1}^{4} D_{i} } \\
= & \left(J_{A}+M_{A}\right) \frac{\delta}{\delta \Phi_{A}^{*}}\left(J_{B}+M_{B}\right) \frac{\delta}{\delta \Phi_{B}^{*}}-\frac{\mathrm{i}}{\hbar}\left(J_{A}+M_{A}\right) \frac{\delta}{\delta \Phi_{A}^{*}} J_{B} M^{B *} \\
& -\frac{\mathrm{i}}{\hbar} J_{B} M^{B *}\left(J_{A}+M_{A}\right) \frac{\delta}{\delta \Phi_{A}^{*}}+\left(\frac{\mathrm{i}}{\hbar}\right)^{2} J_{A} M^{A *} J_{B} M^{B *} .
\end{aligned}
$$

After rearranging the antifield derivatives, the four summands in (A.2) take the form

$$
\begin{aligned}
& D_{1}=(-1)^{\varepsilon_{A}+1}\left(J_{A}+M_{A}\right)\left(J_{B}+M_{B}\right) \frac{\delta}{\delta \Phi_{B}^{*}} \frac{\delta}{\delta \Phi_{A}^{*}}+\left(J_{A}+M_{A}\right) \frac{\delta M_{B}}{\delta \Phi_{A}^{*}} \frac{\delta}{\delta \Phi_{B}^{*}} \\
& D_{2}=(-1)^{\varepsilon_{A}} \frac{\mathrm{i}}{\hbar}\left(J_{A}+M_{A}\right) J_{B} M^{B *} \frac{\delta}{\delta \Phi_{A}^{*}}-(-1)^{\varepsilon_{B}\left(\varepsilon_{A}+1\right)} \frac{\mathrm{i}}{\hbar}\left(J_{A}+M_{A}\right) J_{B} \frac{\delta M^{B *}}{\delta \Phi_{A}^{*}} \\
& D_{3}=-\frac{\mathrm{i}}{\hbar} J_{B} M^{B *}\left(J_{A}+M_{A}\right) \frac{\delta}{\delta \Phi_{A}^{*}} \\
& D_{4}=(-1)^{\varepsilon_{B}}\left(\frac{\mathrm{i}}{\hbar}\right)^{2} J_{B} J_{A} M^{A *} M^{B *}+\frac{\mathrm{i}}{\hbar} J_{A} M_{B}^{A *} M^{B *}
\end{aligned}
$$

where the notation

$$
M_{B}^{A *}=\left.\frac{\delta^{2} M\left(\Phi, \Phi^{*}\right)}{\delta \Phi_{A}^{*} \delta \Phi^{B}}\right|_{\Phi \rightarrow \frac{\hbar}{\mathrm{i}} \frac{\delta}{\delta J}}
$$

was used.

The first term in $D_{4}$ vanishes identically, whereas the first one in $D_{1}$ reads

$$
(-1)^{\varepsilon_{A}+1}\left(J_{A} J_{B}+M_{A} M_{B}+J_{A} M_{B}+(-1)^{\varepsilon_{A} \varepsilon_{B}} J_{B} M_{A}+\frac{\hbar}{\mathrm{i}} M_{A B}\right) \frac{\delta}{\delta \Phi_{B}^{*}} \frac{\delta}{\delta \Phi_{A}^{*}},
$$

with

$$
M_{A B}=\left.\frac{\delta^{2} M\left(\Phi, \Phi^{*}\right)}{\delta \Phi^{A} \delta \Phi^{B}}\right|_{\Phi \rightarrow \frac{\hbar}{\mathrm{i}} \frac{\delta}{\delta J}} \quad \text { so that } \quad M_{A B}=(-1)^{\varepsilon_{A} \varepsilon_{B}} M_{B A} .
$$

Since under the exchange $A \leftrightarrow B$ the symmetry property of the expression in brackets is opposite to the symmetry of the second antifield derivative, (A.5) vanishes, and $D_{1}$ is reduced to the second term.

Next, we collect the remaining terms in (A.3) which are not proportional to an antifield derivative operator, i.e. the second terms in $D_{2}$ and $D_{4}$,

$$
\frac{\mathrm{i}}{\hbar}\left[J_{A}\left(M_{B}^{A_{B}^{*}} M^{B *}-M_{B} \frac{\delta M^{A *}}{\delta \Phi_{B}^{*}}(-1)^{\varepsilon_{A}}\right)-\left(J_{A} J_{B}+\frac{\hbar}{\mathrm{i}} M_{A B}\right) \frac{\delta M^{A *}}{\delta \Phi_{B}^{*}}(-1)^{\varepsilon_{A}}\right] .
$$


Note that

$$
\frac{\delta M^{A *}}{\delta \Phi_{B}^{*}}=\left.\frac{\delta^{2} M\left(\Phi, \Phi^{*}\right)}{\delta \Phi_{B}^{*} \delta \Phi_{A}^{*}}\right|_{\Phi \rightarrow \frac{\hbar}{\mathrm{i}} \frac{\delta}{\delta J}} \quad \text { and } \quad \frac{\delta M^{A *}}{\delta \Phi_{B}^{*}}=\frac{\delta M^{B *}}{\delta \Phi_{A}^{*}}(-1)^{\left(\varepsilon_{A}+1\right)\left(\varepsilon_{B}+1\right)}
$$

and, therefore,

$$
\left(J_{A} J_{B}+\frac{\hbar}{\mathrm{i}} M_{A B}\right) \frac{\delta M^{A *}}{\delta \Phi_{B}^{*}}(-1)^{\varepsilon_{A}}=0
$$

due to symmetry properties under $A \leftrightarrow B$. From $(2.12),(M, M)=0$, we have

$$
0=\frac{1}{2} \frac{\delta}{\delta \Phi_{A}^{*}}(M, M)=\frac{\delta^{2} M\left(\Phi, \Phi^{*}\right)}{\delta \Phi_{A}^{*} \delta \Phi_{B}} \frac{\delta M\left(\Phi, \Phi^{*}\right)}{\delta \Phi_{B}^{*}}-\frac{\delta M\left(\Phi, \Phi^{*}\right)}{\delta \Phi^{B}} \frac{\delta^{2} M\left(\Phi, \Phi^{*}\right)}{\delta \Phi_{B}^{*} \delta \Phi_{A}^{*}}(-1)^{\varepsilon_{A}}
$$

which, after substituting $\Phi \rightarrow \frac{\hbar}{\mathrm{i}} \frac{\delta}{\delta J}$, yields

$$
M_{B}^{A *} M^{B *}-M_{B} \frac{\delta M^{A *}}{\delta \Phi_{B}^{*}}(-1)^{\varepsilon_{A}}=0 .
$$

We have thus shown that the expression (A.7) vanishes.

Finally, the terms in (A.3) proportional to a single antifield derivative, i.e. the second term in $D_{1}$, the first one in $D_{2}$ and all of $D_{3}$, have the form

$$
\begin{aligned}
& \left(J_{A}+M_{A}\right) \frac{\delta M_{B}}{\delta \Phi_{A}^{*}} \frac{\delta}{\delta \Phi_{B}^{*}}+\frac{\mathrm{i}}{\hbar}\left[(-1)^{\varepsilon_{A}}\left(J_{A}+M_{A}\right) J_{B} M^{B *}-J_{B} M^{B *}\left(J_{A}+M_{A}\right)\right] \frac{\delta}{\delta \Phi_{A}^{*}} \\
& =\left(M_{A} \frac{\delta M_{B}}{\delta \Phi_{A}^{*}}+M_{B A} M^{A *}(-1)^{\varepsilon_{B}}\right) \frac{\delta}{\delta \Phi_{B}^{*}} .
\end{aligned}
$$

Again, this expression is equal to zero as a consequence from the analog of the classical master equation $(M, M)=0$. Indeed,

$$
0=\frac{1}{2} \frac{\delta}{\delta \Phi^{A}}(M, M)=\frac{\delta M\left(\Phi, \Phi^{*}\right)}{\delta \Phi_{B}} \frac{\delta^{2} M\left(\Phi, \Phi^{*}\right)}{\delta \Phi_{B}^{*} \delta \Phi^{A}}+(-1)^{\varepsilon_{A}} \frac{\delta^{2} M\left(\Phi, \Phi^{*}\right)}{\delta \Phi^{A} \delta \Phi^{B}} \frac{\delta M\left(\Phi, \Phi^{*}\right)}{\delta \Phi_{B}^{*}}
$$

thus substituting $\Phi \rightarrow \frac{\hbar}{\mathrm{i}} \frac{\delta}{\delta J}$ we find

$$
M_{B} \frac{\delta M_{A}}{\delta \Phi_{B}^{*}}+(-1)^{\varepsilon_{A}} M_{A B} M^{B *}=0 .
$$

We have proved our assertion that $\hat{q}^{2}=0$.

\section{References}

[1] C. Becchi, A. Rouet and R. Stora, Renormalization of the abelian Higgs-Kibble model, Commun. Math. Phys. 42 (1975) 127 [SPIRES].

[2] I.V. Tyutin, Gauge invariance in field theory and statistical physics in operator formalism, arXiv: 0812.0580 [SPIRES].

[3] L.D. Faddeev and A.A. Slavnov, Gauge fields: Introduction to quantum theory, Benjamin/Cummings (1980). 
[4] M. Henneaux and C. Teitelboim, Quantization of gauge systems, Princeton University Press, Princeton U.S.A. (1992).

[5] S. Weinberg, The quantum theory of fields, Vol. II, Cambridge University Press, Cambridge U.K. (1996).

[6] D.M. Gitman and I.V. Tyutin, Quantization of fields with constraints, Springer (1990).

[7] M.A.L. Capri et al., A remark on the BRST symmetry in the Gribov-Zwanziger theory, Phys. Rev. D 82 (2010) 105019 [arXiv:1009.4135] [SPIRES].

[8] L. Baulieu et al., Renormalizability of a quark-gluon model with soft BRST breaking in the infrared region, Eur. Phys. J. C 66 (2010) 451 [arXiv:0901.3158] [SPIRES].

[9] D. Dudal, S.P. Sorella, N. Vandersickel and H. Verschelde, Gribov no-pole condition, Zwanziger horizon function, Kugo-Ojima confinement criterion, boundary conditions, BRST breaking and all that, Phys. Rev. D 79 (2009) 121701 [arXiv:0904.0641] [SPIRES].

[10] L. Baulieu and S.P. Sorella, Soft breaking of BRST invariance for introducing non-perturbative infrared effects in a local and renormalizable way, Phys. Lett. B 671 (2009) 481 [arXiv:0808.1356] [SPIRES].

[11] M.A.L. Capri et al., Renormalizability of the linearly broken formulation of the BRST symmetry in presence of the Gribov horizon in Landau gauge Euclidean Yang-Mills theories, Phys. Rev. D 83 (2011) 105001 [arXiv:1102.5695] [SPIRES].

[12] D. Dudal, S.P. Sorella and N. Vandersickel, The dynamical origin of the refinement of the Gribov-Zwanziger theory, arXiv:1105.3371 [SPIRES].

[13] D. Zwanziger, Action from the Gribov horizon, Nucl. Phys. B 321 (1989) 591 [SPIRES].

[14] D. Zwanziger, Local and renormalizable action from the Gribov horizon, Nucl. Phys. B 323 (1989) 513 [SPIRES].

[15] V.N. Gribov, Quantization of nonabelian gauge theories, Nucl. Phys. B 139 (1978) 1 [SPIRES].

[16] P.M. Lavrov and I.V. Tyutin. On the structure of renormalization in gauge theories (in Russian), Sov. J. Nucl. Phys. 34 (1981) 156 [Yad. Fiz. 34 (1981) 277] [SPIRES].

[17] P.M. Lavrov and I.V. Tyutin. On the generating functional for the vertex functions in Yang-Mills theories (in Russian), Sov. J. Nucl. Phys. 34 (1981) 474 [Yad. Fiz. 34 (1981) 850] [SPIRES].

[18] I.A. Batalin and G.A. Vilkovisky, Gauge algebra and quantization, Phys. Lett. B 102 (1981) 27 [SPIRES].

[19] I.A. Batalin and G.A. Vilkovisky, Quantization of gauge theories with linearly dependent generators, Phys. Rev. D 28 (1983) 2567 [SPIRES].

[20] B.S. DeWitt, Dynamical theory of groups and fields, Gordon and Breach, New York U.S.A. (1965).

[21] G. Leibbrandt, Introduction to the technique of the dimensional regularization, Rev. Mod. Phys. 47 (1975) 849 [SPIRES].

[22] B.L. Voronov, P.M. Lavrov and I.V. Tyutin, Canonical transformations and gauge dependence in general gauge theories (in Russian), Sov. J. Nucl. Phys. 36 (1982) 292 [Yad. Fiz. 36 (1982) 498] [SPIRES].

[23] R.F. Sobreiro and S.P. Sorella, A study of the Gribov copies in linear covariant gauges in Euclidean Yang-Mills theories, JHEP 06 (2005) 054 [arXiv:0506165] [SPIRES]. 